\title{
Constipación funcional en pediatría: Criterios de Roma IV, diagnóstico y tratamiento
}

\section{Functional constipation in paediatrics: The Rome IV criteria, diagnosis, and treatment.}

Avelar-Rodríguez $\mathrm{D}^{1}$, Toro-Monjaraz EM², Ramírez-Mayans JA

\section{INTRODUCCIÓN}

La constipación (o estreñimiento) en pediatría es una de las entidades pediátricas más comunes que el médico general y el pediatra ven en la práctica diaria. Es la causa gastrointestinal más común que resulta en la derivación del paciente a especialistas, causa significativa de ansiedad en los padres. La constipación en pediatría es funcional en 90-95\% de los casos. El diagnóstico continúa siendo basado en los criterios de Roma IV. El tratamiento consiste en una etapa de desimpactación y una de mantenimiento. ${ }^{1-3}$

\section{ETIOLOGÍA Y FISIOPATOLOGÍA}

La constipación es una entidad clínica caracterizada por una disminución de la frecuencia de las evacuaciones, retención de heces, evacuaciones dolorosas o duras, heces de gran tamaño e incontinencia fecal. Múltiples causas orgánicas han sido descritas; sin embargo, la causa más común es funcional (i.e. no existe evidencia de una etiología orgánica), lo cual corresponde a un $90-95 \%$ de los pacientes con constipación. La constipación funcional es un comportamiento o instinto aprendido causado principalmente por evacuaciones dolorosas y factores sociales. El paso de una evacuación dura y dolorosa genera miedo en el niño, provocando el instinto de retener las evacuaciones y un "círculo vicioso" (Figura 1). La constipación funcional es un
${ }^{1}$ Pasante del Servicio Social, Universidad Autónoma de Guadalajara.

${ }^{2}$ Adscrito al Servicio de Gastroenterología y a la Unidad de Motilidad y Fisiología Gastrointestinal, Instituto Nacional de Pediatría.

${ }^{3}$ Jefe del Depto. de Gastroenterología, Instituto Nacional de Pediatría.

Recibido: 5 de octubre del 2017

Aceptado: 21 de noviembre del 2017

Correspondencia

David Avelar Rodríguez

davidavelar1@outlook.com

Este artículo debe citarse como

Avelar-Rodríguez D, Toro-Monjaraz EM, RamírezMayans JA. Constipación funcional en pediatría: Criterios de Roma IV, diagnóstico y tratamiento. Acta Pediatr Mex. 2018;39(1):81-84. 


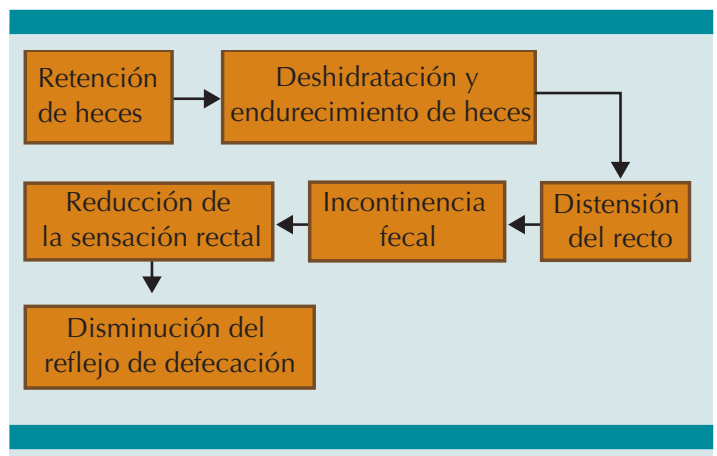

Figura 1. Muestra la fisiopatología ("circulo vicioso") de la constipación funcional.

problema complejo y comprende una variedad de factores contribuyentes (modelo conceptual psicosocial) como son: anormalidades de la motilidad gastrointestinal; hipersensibilidad visceral; problemas psicológicos; microbiota intestinal alterada; eventos traumáticos; evacuaciones dolorosas; cambios en la dieta, entre otros. $^{4-7}$

\section{EPIDEMIOLOGÍA}

Una revisión sistemática estimó la prevalencia mundial de constipación en pacientes pediátricos de un 0.7 a $29.6 \%$ (con una media del $12 \%$ ), y también encontraron una predominancia discreta del sexo femenino, con una relación de 1.2:1. ${ }^{8}$ En el primer año de vida, su prevalencia es de un $2.9 \%$ y en el segundo año de vida es de un $10.1 \% .^{9}$

\section{DIAGNÓSTICO}

La historia clínica y la exploración física de calidad son cruciales para excluir causas orgánicas, diagnosticar constipación funcional y para reconocer complicaciones. Es de gran importancia saber la frecuencia fisiológica de las evacuaciones en los diferentes grupos de edad, ya que sin el conocimiento de estos se puede confundir con una disminución en las evacuaciones, lo que conllevaría a tratamientos innecesarios. La frecuencia normal de las evacuaciones disminuye conforme la edad avanza: de 0 a 3 meses, 3 evacuaciones por día en niños alimentados con leche materna, y 2 en niños alimentados con formula; de 6 a 12 meses, 2 por día; de 1 a 3 años, 1.4 por día; y finalmente, niños mayores de 3 años, 1 por día. ${ }^{10}$

El diagnóstico de constipación funcional es basado en los criterios de Roma IV (Cuadros 1 y 2 ). Las diferencias entre los criterios de Roma III y Roma IV para constipación son los siguientes: 1)

Cuadro 1. Criterios de Roma IV para el diagnóstico de constipación funcional en niños mayores de 4 años $^{6}$

Dos o más criterios, los cuales deben estar presentes por lo menos $1 \mathrm{vez} / \mathrm{semana}$ por un mínimo de 1 mes, con insuficientes criterios para el diagnóstico de síndrome de intestino irritable.

1.Dos o menos evacuaciones/semana en un niño con edad de 4 años en adelante.

2. Por lo menos 1 episodio de inconstancia fecal/semana.

3. Antecedente de posturas de retención.

4. Antecedente de evacuaciones dolorosas o heces duras.

5. Presencia de masa fecal en el recto.

6 . Antecedente de heces de gran diámetro que obstruyen el inodoro.

Después de una evaluación exhaustiva, los síntomas no pueden ser atribuidos a otra condición médica.

Cuadro 2. Criterios de Roma IV para el diagnóstico de constipación funcional en niños menores de 4 años $^{9}$

Dos o más criterios, los cuales deben de estar presentes por lo menos $1 \mathrm{vez} / \mathrm{mes}$ en un paciente menor de 4 años.

1. Dos o menos defecaciones/semanas.

2. Antecedente de retención excesiva de heces.

3. Antecedente de evacuaciones dolorosas o duras.

4. Antecedente de heces de gran tamaño.

5. Presencia de masa fecal en el recto.

En niños que ya no usan pañal, los siguientes criterios pueden aplicar:

6. Por lo menos 1 episodio de incontinencia fecal/semana después de haber dejado el pañal.

7. Antecedente de heces de gran tamaño que obstruyen el inodoro. 
Para niños mayores de 4 años, la duración de los síntomas para el diagnóstico cambió de 2 meses a 1 mes, esto para evitar un retraso en el inicio del tratamiento; 2) Para niños menores de 4 años, se definieron nuevos criterios para niños que no usan pañal, y el síntoma de "incontinencia fecal" se movió a los "criterios adicionales para niños que no usan pañal", ya que el reconocimiento de la incontinencia fecal en pacientes que usan pañal no es confiable. ${ }^{6,9}$

Las posturas de retención requieren especial atención, ya que con frecuencia los padres lo confunden con la acción del niño de estar "pujando" para poder evacuar; sin embargo, éstas no son más que el intento del niño de evadir la evacuación por medio de la contracción del esfínter anal exterior y los músculos glúteos - típicamente, el niño se esconde en una esquina y balancea su cuerpo hacia delante y hacia atrás. ${ }^{11}$

Aunque el uso del tacto rectal es controversial, y algunas referencias no lo recomiendan por su bajo valor clínico (Likelihood Ratio de 1.2), ${ }^{12}$ los criterios de Roma IV y las guías del National Institute for Health and Care Excellence (NICE) recomiendan su uso, en casos en los que el paciente no cumpla con todos los criterios, o que el médico tenga sospecha de una etiología orgánica. ${ }^{6,13}$ De igual manera, la presencia de impactación fecal (incontinencia fecal, masa abdominal palpable, o por medio de tacto rectal) es importante para guiar el abordaje terapéutico. Sin embargo, la radiografía abdominal no se recomienda, ya que la interpretación del contenido fecal en el recto varía significativamente entre los diferentes radiólogos. El uso rutinario de exámenes de laboratorio tampoco es recomendado, con la única excepción de que se tenga sospecha de una etiología orgánica. 6,11-13 Por tal motivo, el diagnóstico de constipación funcional es meramente clínico y basado en los criterios de Roma IV.
La minoría de los pacientes (5-10\%) en quienes existe un alto índice de sospecha de una constipación de origen orgánico deberán de ser referidos al pediatra gastroenterólogo. Los signos de alarma son: inicio de los síntomas antes de 1 mes de edad; sangre en heces sin fisura anal; expulsión de meconio tardía (>48 horas); vomito biliar; detención del crecimiento; panel tiroideo anormal; distensión abdominal grave; fístula perianal; reflejo cremastérico o de extremidades inferiores ausente o disminuido; sospecha de espina bífida oculta; miedo extremo ante la inspección anal o cicatrices anales. ${ }^{11,13}$

\section{TRATAMIENTO}

La finalidad del manejo consiste en lograr evacuaciones de frecuencia y consistencia normales (basado en la escala de Bristol, y en la frecuencia de evacuaciones ya antes mencionadas). Además del tratamiento farmacológico, se deberá recomendar una dieta rica en frutas y fibra, una ingesta importante de agua, y un incremento en la actividad física; ${ }^{1,5}$ la evidencia es controversial, sin embargo, es importante promover estos hábitos en niños con estreñimiento. ${ }^{12}$ Igualmente, en niños que ya van al baño se recomienda que se sienten en el inodoro después de la comida hasta 5 minutos, y con el apoyo de un banquito para promover el pujo. ${ }^{1}$

El manejo farmacológico se puede dividir en dos etapas: la etapa de desimpactación, y la de mantenimiento. La primera se puede realizar en casa por parte de los familiares. El polietilenglicol 3350 (PEG 3350) se inicia a una dosis de 1-1.5 g/kg/día, sin embargo, la dosis es guiada por la dosis respuesta de la consistencia y la frecuencia de las heces. Esta etapa puede tener una duración de 2-3 días, dependiendo de cada paciente. El PEG 3350 es igualmente efectivo que los enemas para la etapa de desimpactación, aunque se recomienda iniciar con la terapia oral. 
Existen varios laxantes osmóticos orales para el tratamiento de mantenimiento como son el PEG 3350 (0.2-0.8 g/ kg/día), la lactulosa (1-2 g/ kg una o dos veces/día), y la leche de magnesia (2-5 años: 0.4-1.2 g/día; 6-11 años: 1.2-2.4 g/ día; 12-18 años: 2.4-4.8 g/día). Como también los laxantes estimulantes como el bisacodilo (3-10 años: $5 \mathrm{mg} / \mathrm{día}$; >10 años: 5-10 mg/día), senosidos (2-6 años: 2.5-5 mg 1-2 veces/día; 6-12 años: 7.5-10 mg/día; >12 años: 15-20 $\mathrm{mg} / \mathrm{día})$, y el picosulfato sódico (1 mes-4 años: 2.5-10 mg/día; 4-18 años: 2.5-20 mg/día); sin embargo, el PEG 3350 es el más efectivo. (Es esencial primero resolver la impactación fecal para poder iniciar la etapa de mantenimiento). Es importante tener en cuenta que las dosis sirven como guía, pero la dosificación será guiada por la dosis-respuesta de la consistencia y la frecuencia de las heces. A la fecha, la duración de la terapia de mantenimiento no ha sido bien establecida por la falta de estudios aleatorizados; sin embargo, es importante mencionar que no se debe suspender el tratamiento de forma inmediata una vez el paciente comienza a evacuar normal y es recomendado que se continúe por al menos 2 meses. ${ }^{1,4,11-15}$

\section{REFERENCIAS}

1. Auth MK, Vora R, Farrelly P, Baillie C. Childhood constipation. BMJ. 2012 Nov 13; 345:e7309.

2. Xinias I, Mavroudi A. Constipation in Childhood. An update on evaluation and management. Hippokratia. 2015;19(1):11-9.

3. Rajindrajith S, Devanarayana NM, Crispus Perera BJ, Benninga MA. Childhood constipation as an emerging public health problem. World J Gastroenterol. 2016;22(30):6864-75.

4. Drossman DA. Functional Gastrointestinal Disorders: History, Pathophysiology, Clinical Features and Rome IV. Gastroenterology. 2016 Feb 19. pii:S0016-5085(16)00223-7.
5. Howarth LJ, Sullivan PB. Management of chronic constipation in children, Paediatrics and Child Health (2016). DOI: http://dx.doi.org/10.1016/j.paed.2016.06.007

6. Hyams JS, Di Lorenzo C, Saps M, Shulman RJ, Staiano A, van Tilburg M. Functional Disorders: Children and Adolescents. Gastroenterology. 2016 Feb 15.pii: S00165085(16)00181-5.

7. Poddar U. Approach to Constipation in Children. Indian Pediatr. 2016;53(4):319-27.

8. Mugie SM, Benninga MA, Di Lorenzo C. Epidemiology of constipation in children and adults: a systematic review. Best Pract Res Clin Gastroenterol. 2011;25(1):3-18.

9. Benninga MA, Faure C, Hyman PE, St James Roberts I, Schechter NL, Nurko S. Childhood Functional Gastrointestinal Disorders: Neonate/Toddler. Gastroenterology. 2016 Feb 15. pii: S0016-5085(16)00182-7.

10. José María Remes Troche, José Antonio Chávez Barrera, Beatriz González Ortíz, et al. Guías de diagnóstico y tratamiento del estreñimiento en México. D) Evaluación y tratamiento del estreñimiento en población pediátrica. Revista de Gastroenterología de Mexico. https://www.gastro.org.mx/wp-content/uploads/2014/06/estrenimientoevaluacion.pdf

11. Nurko S, Zimmerman LA. Evaluation and treatment of constipation in children and adolescents. Am Fam Physician. 2014;90(2):82-90.

12. Tabbers MM, DiLorenzo $C$, Berger MY, Faure $C$, Langendam MW, Nurko S, Staiano A, Vandenplas $Y$, Benninga MA; European Society for Pediatric Gastroenterology, Hepatology, and Nutrition; North American Society for Pediatric Gastroenterology. Evaluation and treatment of functional constipation in infants and children: evidence-based recommendations from ESPGHAN and NASPGHAN. J Pediatr Gastroenterol Nutr. 2014;58(2):258-74.

13. Constipation in children and young people: diagnosis and management. National Institute for Health and Care Excellence. Accessed on 14/11/2017 at https://www.nice. org.uk/guidance/cg99

14. Rowan-Legg A; Canadian Paediatric Society, Community Paediatrics Committee. Managing functional constipation in children. Paediatr Child Health. 2011 Dec;16(10):661-70.

15. Phatak UP, Pashankar DS. Role of polyethylene glycol in childhood constipation. Clin Pediatr (Phila). 2014;53(10):927-32. 